\title{
ON LYAPUNOV TYPE FINITE DIFFERENCE INEQUALITY
}

\author{
B. G. PACHPATTE
}

\begin{abstract}
Lyapunov type finite difference inequality is established which in the special case yields implicit lower bound on the distance between consecutive zeros of a nontrivial solution of a second order linear finite difference equation.
\end{abstract}

The classical inequality of Lyapunov [5] states that if $y(t)$ is a nontrival solution of the second order differential equation

$$
y^{\prime \prime}+p(t) y=0
$$

where $p(t)$ is real and continuous, and if $y(t)$ has at least two zeros on the interval [a,b], then

$$
(b-a) \int_{a}^{b}|p(t)| d t>4 .
$$

Inequality (2) provides an implicit lower bound on the distance between the zeros of a nontrivial solution of (1) by means of an integral measurement of $p$. The Lyapunov inequality has received considerable attention since its appearance and a number of papers have been appeared in the literature which deals with the various extensions, generalizations and applications of this inequality, see [1-8] and the references given therein.

The main purpose of this note is to establish a Lyapunov type inequality for the second order linear finite difference equation

$$
\Delta(r(n) \Delta x(n))+p(n) x(n)=0,
$$

for $n \in I$, where $I=\{a, a+1, a+2, \ldots, b\}, a$ and $b=a+m,(m \geq 2)$ integers, the operator $\Delta$ is defined by $\Delta z(n)=z(n+1)-z(n)$ for $n \in I$. It is assumed that $p(n)$ and $r(n)$ for $n \in I$ are real-valued functions and $r(n)>0$ for $n \in I$. Here our approach is more direct and elementary and the result provides a new estimate on this type of inequality.

Our main result is established in the following theorem.

Received July 26, 1989. 
Theorem. Let $x(n)$ be a solution of equation (3) such that $x(a)=x(b)=0, x(n) \neq$ 0 , for $n \in I^{0}=\{a+1, a+2, \ldots, b-1\}$. Let $k$ be a point in $I^{0}$ where $|x(n)|$ is maximized. Then

$$
4 \leq\left(\sum_{n=a}^{b-1} \frac{1}{r(n)}\right)\left(\sum_{n=a}^{b-1}|p(n)|\right) .
$$

Proof. Let $M=|x(k)|, k \in I^{0}$. It is obvious that

$$
x(k)=\sum_{n=a}^{k-1} \Delta x(n), \quad k \in I,
$$

and

$$
x(k)=-\sum_{n=k}^{b-1} \triangle x(n), \quad k \in I .
$$

From (5) and (6) we observe that

$$
2 M \leq \sum_{n=a}^{b-1}|\Delta x(n)|
$$

Now squaring both sides of (7) and using the Schwarz inequality, the following formula of summation by parts

$$
\sum_{s=0}^{n-1} u(s) \Delta v(s)=(u(n) v(n)-u(o) v(o))-\sum_{s=0}^{n-1} v(s+1) \Delta u(s)
$$

and the facts that $x(a)=x(b)=0$ and equation (3) we observe that

$$
\begin{aligned}
4 M^{2} & \leq\left(\sum_{n=a}^{b-1} r^{-\frac{1}{2}}(n) r^{\frac{1}{2}}(n)|\Delta x(n)|\right)^{2} \\
& \leq\left(\sum_{n=a}^{b-1} \frac{1}{r(n)}\right)\left(\sum_{n=a}^{b-1}(r(n) \triangle x(n)) \Delta x(n)\right) \\
& =\left(\sum_{n=a}^{b-1} \frac{1}{r(n)}\right)\left(-\sum_{n=a}^{b-1} x(n+1) \triangle(r(n) \Delta x(n))\right) \\
& =\left(\sum_{n=a}^{b-1} \frac{1}{r(n)}\right)\left(\sum_{n=a}^{b-1} x(n+1) p(n) x(n)\right) \\
& \leq\left(\sum_{n=a}^{b-1} \frac{1}{r(n)}\right) M^{2}\left(\sum_{n=a}^{b-1}|p(n)|\right) .
\end{aligned}
$$

Dividing both sides of (9) by $M^{2}$ we get the desired inequality in (4). This completes the proof of Theorem. 
It is interesting to note that in the special case when $r(n)=1$, the inequality established in (4) reduces to the following inequality

$$
4 \leq(b-a) \sum_{n=a}^{b-1}|p(n)| \text {. }
$$

The inequality (10) yields the implicit lower bound on the distance between consecutive zeros of a nontrivial solution of equation (3).

\section{References}

[1] S.B. Eliason, "A Lyapunov inequality for a certain second order nonlinear differential equation", J. London Math. Soc. 2 (1970), 461-466.

[2] A.M. Fink and D.F. St. Mary, "On an inequality of Nehari", Proc. Amer. Math. Soc. 21 (1969), 640-642.

[3] P. Hartman, Ordinary Differential Equations, John Wiley and Sons, New York, 1964.

[4] M.K. Kwong, "On Lyapunov's inequality for disfocality", J. Math. Anal. Appl. 83 (1981), 486-494.

[5] A.M. Liapunov, Problème générale de la stuílitié du mouvement, Annals of Mathematices Study 17, Princeton University Press, 1949.

[6] B.G. Pachpatte, "A note on Lyapunov type inequalities", Indian J. Pure Appl. Math., 21 (1990), 45-49.

[7] W.T. Patula, "On the distance between zeros", Proc. Amer. Math. Soc. 52 (1975), 247-251.

[8] W.T. Reid, "A generalized Liapunov inequality", J. Differential Equations 13 (1973), 182-196.

Department of Mathematics and Statistics, Marathwada University, Aurangabad 431004 (Maharashtra), INDIA. 